\title{
The Mediator Role of Emotion Regulation Processes on Infertility-Related Stress
}

\author{
Ana Galhardo • M. Cunha · J. Pinto-Gouveia • \\ M. Matos
}

(C) Springer Science+Business Media New York 2013

\begin{abstract}
The objective of this study is to investigate gender differences regarding the mediator role of selfcompassion and self-judgment on the effects of external shame, internal shame, dyadic adjustment, on infertilityrelated stress. One hundred and sixty-two women and 147 men with a primary infertility diagnosis completed the following set of self-report measures: Others as Shamer, Experience of Shame Scale, Dyadic Adjustment Scale, SelfCompassion Scale, and Fertility Problem Inventory. Path analyses results revealed that in women self-compassion fully mediated the effect of internal shame on infertilityrelated stress and partially mediated the effect of dyadic adjustment on this variable, while external shame had only a direct effect. In men self-judgment fully mediated the effect of external and internal shame on infertility-related stress. Dyadic adjustment had only a direct effect on infertility-related stress. In conclusion, there is a distinct role of self-compassion and self-judgment on the relationship between shame and infertility-related stress in men and women. Such differences should be taken into account in psychological interventions with these patients. Future research is warranted to further support our results.
\end{abstract}

Keywords Infertility-related stress - External shame · Internal shame $\cdot$ Self-compassion $\cdot$ Self-judgment

\footnotetext{
A. Galhardo $(\bowtie) \cdot$ M. Cunha

Department of Psychology, Instituto Superior Miguel Torga, Largo da Cruz de Celas, ${ }^{\circ}$ 1, 3000-132 Coimbra, Portugal e-mail: anagalhardo@ismt.pt

A. Galhardo · M. Cunha - J. Pinto-Gouveia - M. Matos CINEICC-Faculty of Psychology and Educational Sciences of the University of Coimbra, Coimbra, Portugal
}

\section{Introduction}

Infertility can be defined as a disease of the reproductive system defined by the failure to achieve a clinical pregnancy after 12 months or more of regular unprotected sexual intercourse (Zegers-Hochschild et al., 2009). Previous studies have addressed the psychological impact of the infertility condition as well as the strains associated with its medical treatment in infertile couples (e.g., Chen, Chang, Tsai, \& Juang, 2004; Eugster \& Vingerhoets, 1999; KlonoffCohen \& Natarajan, 2004; Verhaak, Lintsen, Evers, \& Braat, 2010; Verhaak \& Smeenk, 2007; Volgsten, Skoog, Ekselius, Lundkvist, \& Sundstrom, 2010). Some of these couples are able to adjust to infertility but others present problematic emotional responses such as depression and anxiety (Boivin \& Takefman, 1995, 1996; Ramazanzadeh, Noorbala, Abedinia, \& Nazhizadeh, 2009). In fact, infertility may involve several stressful aspects with couples stating that it corresponds to a significant demanding life crisis (Burns \& Covington, 2006; Menning, 1980; Wischmann, Stammer, Scherg, Gerhard, \& Verres, 2001). It is noteworthy that not only do couples face the possibility they may not achieve biological parenthood, but they also have to deal with the unexpected demands of medical treatment.

Although the psychological consequences of infertility have been previously addressed in several studies, to our knowledge, constructs such as external shame, internal shame, dyadic adjustment and their role in infertility-related stress have not been covered. Furthermore, the scarcity of literature integrating emotion regulation processes of self-compassion and self-judgment in the relationship between external shame, internal shame and dyadic adjustment on infertility-related stress, as well as gender differences regarding these relationships, led us to conduct this exploratory study. 
Infertility-related stress can be seen as a complex construct including relatively independent infertility-related domains. Newton, Sherrard, and Glavac (1999) have identified five separate domains: (1) social concern (reminders of infertility, sensitivity to comments of other people, or the feeling of being social isolated from family members or peers); (2) sexual concerns (difficulties in having scheduled sexual relations, or a decrease in sexual pleasure or sexual selfesteem); (3) relationship concern (worries about the impact of infertility on relationship, difficulties in talking about it or accepting/understanding gender differences); (4) need for parenthood (importance of parenthood in the individual's life); and (5) rejection of a childfree lifestyle (negative perception of being childless). Furthermore, previous research has shown that men and women with infertility significantly differ in levels of infertility-related stress (Abbey, Andrews, \& Halman, 1991; Greil, 1997; Moura-Ramos, Gameiro, Canavarro, Soares, \& Santos, 2012), in general, with women scoring higher than men. Several studies have been conducted assessing infertility stress through general measures of anxiety and depression (Chen et al., 2004; Domar, Broome, Zuttermeister, Seibel, \& Friedman, 1992; Eugster \& Vingerhoets, 1999; Mahajan et al., 2010) but these may fail to capture more specific characteristics of the infertility condition such as the ones included in the five domains mentioned above.

Previous studies that investigated the relationship between infertility-related stress and marital adjustment found that higher levels of marital adjustment were related to lower scores in infertility-related stress, as measured by the Fertility Problem Inventory (FPI; Newton et al., 1999). Thus, high marital adjustment can be seen as a protective factor towards infertility-related stress. But research on this topic has produced mixed results. In a study conducted by Guleç, Hassa, Gunes, and Yenilmez (2011), both women and men in the infertility group reported more dyadic adjustment problems than those in the control group. Another study found that women with infertility presented significantly lower scores in dyadic adjustment than control women, but no differences were noted in infertile men (Monga, Alexandrescu, Katz, Stein, \& Ganiats, 2004).

Although these relationships have been addressed in various investigations, the study of other factors impacting on infertility-related stress has mainly focused on coping styles (Peterson, Newton, Rosen, \& Schulman, 2006; Peterson, Pirritano, Block, \& Schmidt, 2011). For example Schmidt, Holstein, Christensen, and Boivin (2005) found that infertile women who were able to attach a positive meaning to their infertility (meaning-based coping strategy) presented less personal, marital and social problems associated with infertility. Another study that investigated coping processes considering the couple as the unit of analysis identified three key coping strategies related to infertility stress, marital adjustment, and depression, namely distancing, self-controlling and accepting responsibility (Peterson et al., 2006).

However, less attention has been given to other constructs such as shame (external and internal), and emotion regulation processes as self-compassion and self-judgment and the role they may have in perceived infertility-related stress. The relationship between shame and emotional difficulties has been well established, particularly regarding depression (for a review see Kim, Thibodeau, \& Jorgensen, 2011) and anxiety (e.g., Irons \& Gilbert, 2005; Tangney, Wagner, \& Gramzow, 1992). This relationship led us to investigate the role of external and internal shame on infertility-related stress. Moreover we were interested in exploring the mediator effect of the emotion regulation processes of self-compassion and self-judgment. Self-compassion has been described as a selfregulation process in terms of dealing with stress because it involves identifying, understanding, and expressing emotions in an adaptive way (Neff, 2003b). It has also been linked to adaptive psychological functioning, as a protective process against self-evaluative anxiety (Neff, Kirkpatrick, \& Rude, 2007). Conversely, self-judgment includes a critical attitude towards the self when facing pain or failure, the feeling of being isolated and separate from others in those instances, and of becoming so immersed in subjective emotion reactions that one is carried away by one's emotions (Neff, 2004). Considering self-judgment as a process in which individuals tend to be self-critical, to feel isolated and disconnected from others, and to overidentify with their negative emotional states (Neff, 2004) we would expect it to be positively associated with infertility-related stress and shame.

Shame can be described as an emotional experience distinguishable in terms of typical thoughts, behaviors and attention focus. In particular, two types of shame have been identified in the literature. External shame is when one's attention is focused on the social and external environment, on how others perceive and feel towards the self. One's shame feelings arise from perceptions that one exists negatively in the mind of others, as inferior, inadequate or flawed (Matos \& Pinto-Gouveia, 2010). On the contrary, internal shame can emerge as a private feeling related to one's own negative personal judgments of one's characteristics, feelings and fantasies (Gilbert, 2002).

Concerning self-compassion, and according to Neff (2004), three major components must be taken into account: self-kindness versus self-judgment, common humanity versus isolation, and mindfulness versus over-identification. Self-compassion corresponds to the summing of self-kindness (warmth and non-judgmental way of relating to oneself in moments of pain, failure or inadequacy), common humanity (the idea that life difficulties, errors and imperfections are all part of the shared human condition), and mindfulness (a receptive mind state of observation of private events as they arise without trying to change or avoid them) 
(Neff, 2003b; Neff et al., 2007). On the contrary, self-judgment entails being harshly self-critical in instances of failure or pain, perceiving one's experiences as separate from the larger human experience and over-identifying with painful thoughts and feelings (Neff, 2003a).

Although scanty, the relevance of these constructs in infertility has received some empirical support (Galhardo, Pinto-Gouveia, Cunha, \& Matos, 2011; Pinto-Gouveia, Galhardo, Cunha, \& Matos, 2012). When compared with fertile controls, depression in patients with infertility was significantly associated with negative emotion processes such as self-judgment and external and internal shame (Galhardo et al., 2011). These patients were also less capable of being in touch in an open and non-judgmental way to their painful inner mental states (e.g. feelings, thoughts, memories) and less capable of perceiving their experiences as part of the larger human experience (PintoGouveia et al., 2012). Based on these findings, the aim of the present study was to examine the role of a comprehensive set of processes (external shame, internal shame, dyadic adjustment, self-compassion, and self-judgment) in the prediction of infertility-related stress. Specifically, the aim was to test whether self-compassion and self-judgment would mediate the effects of external and internal shame and dyadic adjustment on infertility-related stress. To the best of our knowledge, the investigation of these factors in patients with an infertility diagnosis is scarce and information about them may provide the opportunity to gain insight about specific targets for psychological intervention with these couples. Furthermore, previous research has shown that men and women with infertility differ significantly in levels of infertility-related stress (Abbey et al., 1991; Greil, 1997; Moura-Ramos, Gameiro, Canavarro, Soares, et al., 2012b), in general, with women scoring higher than men. For these reasons another aim of the present study was to test the above mentioned relationships in infertile men and women separately.

A better knowledge of these emotion regulation processes in infertile men and women would be a valuable tool to choose psychological interventions that specifically target these emotion regulation processes, such as Acceptance and Commitment Therapy (ACT; Hayes, Stroshal, \& Wilson, 1999) or Compassion Focused Therapy (CFT; Gilbert, 2010a). According to ACT, people cannot choose the emotions they have but can choose how to relate and respond to their private events (e.g., thoughts, feelings, bodily sensations). In this context, emotion regulation processes are not necessarily dysfunctional but they can become rigid and inflexible due to human language and cognition (Blackledge \& Hayes, 2001). Based on this premise, ACT is intended to modify these maladaptive patterns of reacting to inner experiences in order to cultivate a more meaningful and values oriented life. In turn, CFT focuses on developing compassionate skills that enable effective affect regulation (Gilbert, 2005, 2010a, 2010b). This is achieved through the development of a self-to-self relationship based on feelings of compassion, warmth and kindness. The key principles of CFT are: the development of genuine concern for one's wellbeing; learn to be sensitive, sympathetic and tolerant of one's distress; develop deep understanding (empathy) of the roots and causes of one's distress; becoming non-judgmental and less critical of one's self; and developing feelings of warmth toward the self (Gilbert \& Procter, 2006).

\section{Materials and Methods}

\section{Participants}

The sample of this study is composed of 309 patients (162 women and 147 men) with an infertility diagnosis that have looked for treatment in Portuguese infertility public and private clinics in order to achieve parenthood. All participants presented a primary infertility diagnosis (failure to conceive by a couple who has never conceived). These participants were recruited as part of a more comprehensive research investigation of the psychological characteristics of Portuguese persons with infertility. The response rate was of $20 \%$. This low response rate may be related to the fact that there was no direct contact between researchers and respondents. The battery of self-report questionnaires was delivered by medical staff, and this may have had a negative impact on the respondents' motivation to fill out the questionnaires. Although an information sheet explaining the aims of the study was given to all participants, this may have been insufficient to offset the absence of direct contact with researchers. Also, the fact that these self-report instruments assess personal and somewhat intimate information may contribute to the low response rate.

Inclusion criteria were age (18 years or older), and an infertility medical diagnosis. Participants were all married or living with a partner in a heterosexual relationship (requirements of the Portuguese law for the access to Assisted Reproductive Technologies).

Clinical information regarding infertility was provided by the participants (there was no consulting of medical records). The causes of the fertility problems were: $36.6 \%$ female causes (e.g., disorders of ovulation, abnormal fallopian tubes, endometriosis, cervical and uterine disorders, immunological problems); $26.9 \%$ male causes (e.g., abnormality of semen, a history of cryptorchidism, chronic illnesses); $21.7 \%$ both female and male causes; and $14.9 \%$ idiopathic causes. Regarding the duration of fertility problems, participants had been diagnosed for almost 3 years $(M=2.96, S D=2.83)$.The majority of them had already undergone infertility treatments $(72.5 \%)$ and $27.5 \%$ were 
still having pre-treatment tests or waiting for their first treatment cycle. A treatment cycle may include different steps depending on the treatment protocol and needs to be based on individual patient characteristics such as age, treatment efficacy, side-effects, and costs; the main steps for the most frequent protocols (IUI, IVF and ICSI) are presented below. These patients were at various stages of infertility treatment. Concerning the patients' actual treatment status: $28.5 \%$ were in an In Vitro Fertilization (IVF) protocol, which includes ovulation induction, oocyte retrieval, oocyte insemination and embryo transfer to the uterus; $25.2 \%$ in an intracytoplasmic sperm injection (ICSI) protocol, which includes the same steps as the IVF but involves the direct injection of a single sperm into the cytoplasm of an oocyte); $10 \%$ were performing ovarian stimulation as an independent procedure, not included on IVF or ICSI protocols; and $5.2 \%$ were in an Intrauterine Insemination IUI protocol, which involves the introduction of washed sperm directly into the uterus through the cervix by means of a catheter.

\section{Instruments}

A set of self-report measures was selected due to their psychometric characteristics and clinical utility.

Fertility Problem Inventory (FPI; Newton et al., 1999) is a 46-item instrument developed to assess infertility-related stress based on a comprehensive approach. It includes five dimensions: social concern, sexual concern, relationship concern, need for parenthood and rejection of a childfree lifestyle. Social concern is related to reminders of infertility, sensitivity to comments, or the feeling of being social isolated from family members or peers (e.g., "When I see families with children I feel left out"). Sexual concern includes difficulties in having scheduled sexual relations, or a decrease in sexual pleasure or sexual self-esteem (e.g., "I find I've lost my enjoyment of sex because of the fertility problem"). Relationship concern addresses worries about the impact of infertility on relationship, difficulties in talking about it or accepting/understanding gender differences (e.g., "When we try to talk about our fertility problem, it seems to lead to an argument"). Need for parenthood reflects the importance of parenthood in the individual's life (e.g., "I will do just about anything to have a child"). Finally, rejection of a childfree lifestyle is associated with a negative perception of being childless (e.g. "I could visualize a happy life together, without a child"). In this study the Portuguese version, that showed a Cronbach $\alpha$ of .88 (Moura-Ramos, Gameiro, Canavarro, \& Soares, 2012) was used. In the present study the FPI was used as a measure of global infertility-related stress, and a Cronbach $\alpha$ of .90 was found in our sample.

Dyadic Adjustment Scale (DAS; Spanier, 1976) is a 32-item scale designed to measure the overall marital adjustment through a 6-point scale ranging from 0 to 5 . The DAS allows a global score as well as scores on four subscales: satisfaction (e.g., "How often do you discuss or have you considered divorce, separation, or terminating your relationship"), cohesion (e.g., "How often would you say you and your mate work together on a project"), consensus (e.g., "Indicate the extent of agreement or disagreement between you and your partner regarding the amount of time spent together"), and affective expression (e.g., "Indicate if not showing love caused differences of opinions or was a problem in your relationship during the past few weeks"). According to Spanier (1976), the DAS presents an excellent internal consistency (Cronbach $\alpha$ of .96 for the total score). For this study a Portuguese translation by Nobre (2003; unpublished manuscript) was used but the study of psychometric characteristics of the Portuguese version was carried out by Gomez and Leal (2008). These authors confirmed the multidimensionality of the DAS and its good internal consistency (Cronbach $\alpha$ of .90 for the total score). They have also examined its test-retest reliability over a 19-weeks period and found a .75 correlation.

Others as Shamer (OAS; Goss, Gilbert, \& Allan, 1994). This 18 items scale measures external shame (global judgments of how people think others view them). For example, respondents rate on a 5-point Likert scale (0-4) the frequency of their feelings and experiences in items such as "Others see me as empty and unfulfilled" and "I feel other people see me as not good enough". In the study of the original version the authors found this scale to have a Cronbach alpha of .92 and in the Portuguese version (Matos \& Pinto-Gouveia, 2010) a Cronbach $\alpha$ of .91 was reported. In this study OAS also presented a high internal consistency (Cronbach's $\alpha=.93$ ).

Experience of Shame Scale (ESS; Andrews, Qian, \& Valentine, 2002) is a 27 item scale assessing feelings of shame around three key domains of self: character, behaviour, and body. The character subscale has to do with personal habits, manner with others, what sort of person you are and personal ability (typical items for this subscale are "Have you felt ashamed of any of your personal habits?", "Have you tried to conceal from others the sort of person you are?"). The behaviour subscale reflects shame about doing something wrong, saying something stupid and failure in competitive situations (e.g., "Have you tried to cover up or conceal things you felt ashamed of having done?", "Have you felt ashamed when you said something stupid?"). The body subscale is related to feeling ashamed of one's body or parts of it (e.g., "Have you avoided looking at yourself in the mirror?", "Have you wanted to hide or conceal your body or any part of it?"). Each item indicates the frequency of experiencing, thinking and avoiding any of the three areas of shame in the past year and is rated on a 4-point Likert scale (1-4). The Portuguese 
version (Matos \& Pinto-Gouveia, 2010) showed a Cronbach $\alpha$ of .94. In the present study, ESS total showed an excellent internal consistency (Cronbach's $\alpha=.96$ ).

Self-Compassion Scale (SCS; Neff, 2003a) is a measure of self-compassion that includes 26 items rated on a 5-point Likert scale. In this study the Portuguese version (Castilho, Pinto-Gouveia, \& Bento, 2011), showing a Cronbach $\alpha$ of .89 was used. For this study we divided the self-judgment subscale from the self-compassion subscale. The first one corresponds to the sum of self-criticism, isolation and overidentification (e.g., "When I see aspects of myself that I don't like, I get down on myself", "When I fail at something that's important to me, I tend to feel alone in my failure", "When something painful happens I tend to blow the incident out of proportion"), and showed a Cronbach's $\alpha$ of .90 in the current study. The second corresponds to the sum of self-kindness, common humanity, and mindfulness (e.g., "I try to be understanding and patient towards those aspects of my personality I don't like", "I try to see my failings as part of the human condition", "When something upsets me I try to keep my emotions in balance"). The Cronbach $\alpha$ found for this subscale was .92 .

\section{Procedure}

The study was previously approved by Ethical Committees of public centers for infertility treatment, Clinical Directors of private clinics, and by the Portuguese Fertility Association Board.

The participants were contacted by their medical doctors or answered a recruitment announcement posted at the Portuguese Fertility Association (patients' association) website. The purposes of the study and the degree of participation were explained to each patient/couple. Participants were assured that anonymity and confidentiality would be maintained and that they could refuse to participate or withdraw from the study at any time. Once agreed, a consent form was signed by the subjects.

The set of self-report instruments was distributed and it was asked that both partners should answer the questionnaires separately, at home. Participants were requested to return the questionnaires to the research team by mail (postal stationery envelopes were provided for this purpose).

\section{Results}

Statistical Analysis

Data analyses were carried out using SPSS v. 18, and path analyses were estimated in AMOS (v. 18).

Preliminary analysis aimed at investigating gender differences were conducted through the use of independent $\mathrm{t}$ tests and Chi square tests. Correlation analyses were also performed in order to explore relationships between clinical variables and the study variables, as well as the relationships between the variables in men and women separately.

Separate mediational studies for women and men were conducted. We were interested in testing whether self-compassion (SCS_compassion) and self-judgment (SCS_judgment) (mediator variables) mediated the relationship between external shame (OAS), internal shame (ESS) and dyadic adjustment (DAS) (independent, exogenous variables) and infertility-related stress (FPI) (dependent, endogenous variable).

Path analyses were carried out to test for the mediator effects aforementioned. This technique is a special case of structural equation modeling (SEM) and considers hypothetic causal relations between variables that have already been defined. This kind of analysis gives insight on how the predictors are inter-related directly or indirectly to the outcome variable of infertility-related stress. A maximum likelihood method was used to evaluate the significance of regression coefficients. SEM procedure estimates the optimal effect of one set of variables on another set of variables in the same equation, controlling for error (Byrne, 2010; Kline, 2005). Multivariate outliers were screened using Mahalanobis squared distance $\left(D^{2}\right)$ method and uni and multivariate normality was assessed by skewness and kurtosis coefficients. There was no severe violation of normal distribution ( $\mathrm{Sk}<3$ and $\mathrm{Ku}<8-10$; Kline, 2005).The significance of direct, indirect and total effects was assessed using $\chi^{2}$ tests (Kline, 2005). The significance of the meditational paths was further tested using Bootstrapping procedure (1,000 bootstrap samples and $95 \%$ confidence intervals (CIs)). This is a statistical resampling method to estimate the standard error for unstandardized or standardized total and indirect effects (Kline, 2005).

Effects with $p<.050$ were considered statistically significant.

\section{Sample Characteristics}

Participants in our study present a mean age of 34.51 years old $(S D=4.99)$ and a mean of 14.15 years of education $(S D=3.50)$. They were married or living with their partners for an average of 6 years $(S D=3.56)$.

When considering gender differences, significant differences were found concerning age $\left(t_{(308)}=2.68\right.$, $p=.007)$ and years of education $\left(t_{(308)}=-4.25, p<.001\right)$. When compared to their female partners (age: $M=33.79$, $S D=4.28$ ) (years of education: $M=14.93, S D=3.02$ ), men in our sample were older $(M=35.31, S D=5.58)$ and less educated $(M=13.29, S D=3.79)$. Since our sample was mainly composed by couples, no significant differences were found in years of marriage or cohabitation $\left(t_{(308)}=.29\right.$, $p=.770)$. 


\section{Descriptives}

Gender differences were explored concerning our study variables. As we can see in Table 1, results show that significant differences were found between men and women regarding external shame, internal shame, self-judgment, and infertility-related stress. When compared with their male partners, women presented higher infertility-related stress (women: $M=143.09, S D=31.17$; men: $M=128.19$, $S D=26.91$ ), higher external shame (women: $M=19.92$, $S D=11.43$; men: $M=17.14, S D=10.80)$, higher internal shame (women: $M=52.59, S D=16.47$; men: $M=43.35$, $S D=13.19$ ), and higher scores on self-judgment (women: $M=37.87, S D=9.53$; men: $M=31.21, S D=9.32$ ). With respect to dyadic adjustment $\left(t_{(307)}=1.19, p=.236\right)$ and self-compassion $\left(t_{(307)}=1.01, p=.312\right)$, no significant differences were found.

No significant correlations were found between duration of infertility, diagnostic, or current medical treatment being pursued and the studied variables.

In order to explore the relationship between the variables we performed Pearson product-moment correlations (Table 2) for both genders.

As presented in Table 2, significant correlations were found between some of the variables but these were very low to moderate correlations (Pestana \& Gageiro, 2005). Interestingly, in women self-compassion is significantly and inversely correlated with external and internal shame and infertility-related stress, whereas in men self-compassion only shows an inverse and weak correlation with external shame.

\section{Path Analysis}

Given the proposed hypotheses and gender differences described above, the aim was to test whether self-compassion and self-judgment mediated the effects of external shame, internal shame, and dyadic adjustment on infertilityrelated stress in women and men separately.

The hypothesized model (Fig. 1) was tested through a fully saturated model (i.e. zero degrees of freedom), consisting of 27 parameters. Given that fully saturated models always produce a perfect fit to the data, model fit indices were neither examined nor reported.

In women the model explained $33 \%$ of infertility-related stress variance. In this model the following paths were not statistically significant: the direct effect of external shame on self-compassion $\left(b_{\mathrm{OAS}}=-.131 ; S E b=.072 ; Z=-1.83\right.$; $\left.p=.067 ; \beta_{\mathrm{OAS}}=-.167\right)$, the direct effect of dyadic adjustment on self-judgment $\left(b_{\text {DAS }}=-.057 ; S E b=.041\right.$; $\left.Z=-1.370 ; p=.171 ; \beta_{\text {DAS }}=-.081\right)$, the direct effect of internal shame on infertility-related stress $\left(b_{\mathrm{ESS}}=.230\right.$; $\left.S E b=.186 ; Z=1.236 ; p=.217 ; \beta_{\text {ESS }}=.121\right)$, and the direct effect of self-judgment on infertility-related stress
Table 1 Gender differences regarding external shame (OAS), internal shame (ESS), dyadic adjustment (DAS), selfcompassion (SCS_compassion), self-judgment (SCS_judgment), and infertility-related stress (FPI)

\begin{tabular}{|c|c|c|c|c|c|c|}
\hline & \multicolumn{2}{|c|}{$\operatorname{Men}(n=147)$} & \multicolumn{2}{|c|}{ Women $(n=162)$} & \multirow[t]{2}{*}{$t(307)$} & \multirow[t]{2}{*}{$p$} \\
\hline & $M$ & $S D$ & $M$ & $S D$ & & \\
\hline External shame (OAS) & 17.14 & 10.80 & 19.92 & 11.43 & -2.19 & .029 \\
\hline Internal shame (ESS) & 43.35 & 13.19 & 52.59 & 16.47 & -5.41 & $<.001$ \\
\hline Dyadic adjustment (DAS) & 118.43 & 15.56 & 116.41 & 14.38 & 1.19 & .236 \\
\hline Self-compassion (SCS_compassion) & 40.97 & 8.13 & 39.98 & 8.99 & 1.01 & .312 \\
\hline Self-judgment (SCS_judgment) & 31.21 & 9.32 & 37.87 & 9.53 & -6.20 & $<.001$ \\
\hline Infertility-related stress (FPI) & 128.19 & 26.96 & 143.09 & 31.17 & -4.48 & $<.001$ \\
\hline
\end{tabular}

Table 2 Pearson correlations (two-tailed) between external shame (OAS), internal shame (ESS), dyadic adjustment (DAS), self-compassion (SCS_compassion), self-judgment (SCS_judgment), and infertility-related stress (FPI) in men and women

\begin{tabular}{|c|c|c|c|c|c|c|}
\hline & OAS & ESS & DAS & SCS_compassion & SCS_judgment & FPI \\
\hline External shame (OAS) & & $.56^{* *}$ & $-.39 * *$ & $-.21 *$ & $.60 * *$ & $.28 * *$ \\
\hline Internal shame (ESS) & $.68 * *$ & & $-.29 * *$ & -.06 & $.52 * *$ & $.29 * *$ \\
\hline Dyadic adjustment (DAS) & $-.35^{* *}$ & $-.33^{* *}$ & & .12 & $-.29 * *$ & $-.35^{* *}$ \\
\hline Self-compassion (SCS_compassion) & $-.46^{* *}$ & $-.51 * *$ & $.34 * *$ & & $-.17 *$ & -.11 \\
\hline Self-judgment (SCS_judgment) & $.58 * *$ & $.65 * *$ & $-.32 * *$ & $-.66^{* *}$ & & $.35^{* *}$ \\
\hline Infertility-related stress (FPI) & $.47 * *$ & $.45 * *$ & -.38 & $-.44 * *$ & $.43^{* *}$ & \\
\hline
\end{tabular}

Intercorrelations for male participants $(n=147)$ are presented above the diagonal, and intercorrelations for female participants $(n=162)$ are presented below the diagonal in italics

$* * p<.010, * p<.050$ 


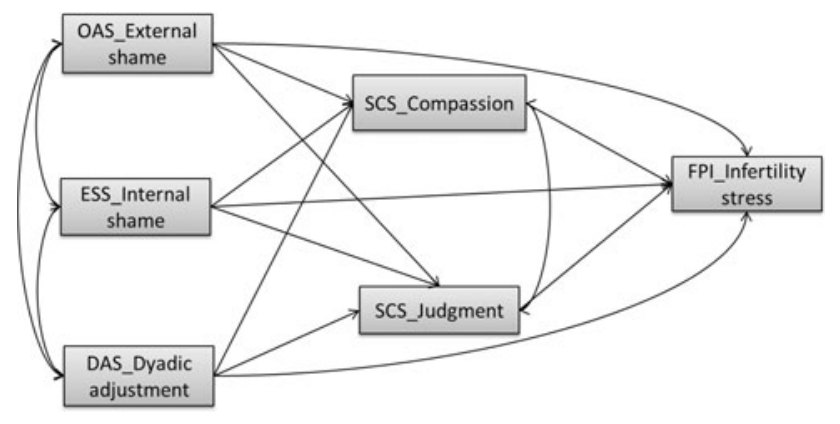

Fig. 1 The theoretical model. Self-compassion (SCS_compassion) and self-judgment (SCS_Judgment) mediate the effect of external shame (OAS), internal shame (ESS) and dyadic adjustment (DAS) upon infertility-related stress (FPI)

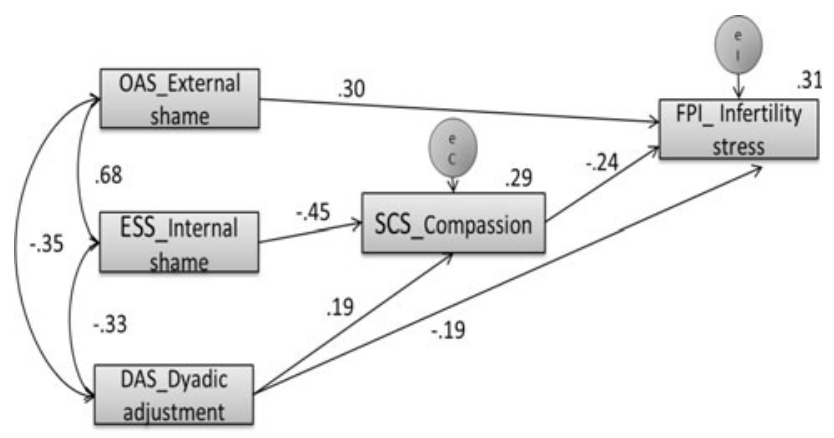

Fig. 2 Results of mediation path analysis showing the relationships among external shame (OAS), internal shame (ESS), dyadic adjustment (DAS) and infertility-related stress (FPI), having self-compassion (SCS_Compassion) as mediator in women, with standardised estimates and square multiple correlations $(n=162)$

$\left(b_{\text {SCS_judgment }}=.104 ; S E b=.328 ; Z=.317 ; p=.751 ;\right.$ $\beta_{\text {SCS_judgment }}=.032$ ).

For this reason, the non-significant paths were removed, self-judgment variable was excluded and the model recalculated (Fig. 2). In the evaluation of the final adjusted model, an excellent model fit was found with a non-significant Chi square of $5.310(\mathrm{df}=2, p=.070)$. Besides, the analysis of well-known and recommended goodness of fit indices (Kline, $2005)$ indicated a very good model fit $(\mathrm{CMIN} / \mathrm{DF}=2.655$; $\mathrm{CFI}=.986 ; \mathrm{TLI}=.930 ; \mathrm{NFI}=.978 ; \quad \mathrm{RMSEA}=.101$; SRMR $=.028)$. All the paths were statistically significant and the significance of indirect mediational paths was further confirmed using bootstrap resampling method. The model accounted for $31 \%$ of infertility-related stress and $29 \%$ of self-compassion variances.

Indirect mediational test results indicated that external shame (OAS) predicted greater infertility-related stress directly with an effect of .30. Internal shame (ESS) predicted elevated infertility-related stress fully through diminished self-compassion (SCS_compassion) $\quad\left(b_{\mathrm{ESS}}=.11,95 \%\right.$ $\mathrm{CI}=.048-.187)$. Dyadic adjustment predicted diminished infertility-related stress partially through increased selfcompassion $\left(b_{\mathrm{DAS}}=-.05,95 \% \mathrm{CI}=-.102\right.$ to -.016$)$, but also revealed a significant direct effect of -.190 .

To summarize, in women self-compassion fully mediated the effect of internal shame on infertility-related stress and partially mediated the effect of dyadic adjustment on this variable, while external shame had only a direct effect.

In men the model explained $20 \%$ of infertility-related stress variance. In this model the following paths were not statistically significant: the direct effect of internal shame on self-compassion $\left(b_{\mathrm{ESS}}=.055 ; S E b=.060 ; Z=.945\right.$; $\left.p=.360 ; \quad \beta_{\mathrm{ESS}}=.089\right)$, the direct effect of dyadic adjustment on self-judgment $\left(b_{\mathrm{DAS}}=-.028 ; S E b=.041\right.$; $\left.Z=-.684 ; p=.494 ; \beta_{\mathrm{DAS}}=-.047\right)$, and on self-compassion $\left(b_{\mathrm{DAS}}=.031 ; S E b=.046 ; Z=.667 ; p=.504\right.$; $\left.\beta_{\mathrm{DAS}}=.059\right)$, the direct effect of external shame on infertility-related stress $\quad\left(b_{\mathrm{OAS}}=-.041 ; \quad S E b=.256\right.$; $\left.Z=-.158 ; p=.874 ; \beta_{\mathrm{OAS}}=-.016\right)$, the direct effect of internal shame on infertility-related stress $\left(b_{\mathrm{ESS}}=.206\right.$; $\left.S E b=.191 ; Z=1.078 ; p=.281 ; \beta_{\mathrm{ESS}}=.101\right)$, and the direct effect of self-compassion on infertility-related stress $\left(b_{\text {SCS_compassion }}=-.114 ; \quad S E b=.253 ; \quad Z=-.452\right.$; $\left.p=.651 ; \beta_{\text {SCS_compassion }}=-.035\right)$.

The non-significant paths were then removed, self-compassion variable was excluded and the model recalculated (Fig. 3). In the evaluation of the final adjusted model, an excellent model fit was found with a non-significant Chi square of $1.610(\mathrm{df}=3, p=.657)$. Besides, the analysis of well-known and recommended goodness of fit indices (Kline, 2005) indicated a very good model fit $(\mathrm{CMIN} / \mathrm{DF}=.537$; $\mathrm{CFI}=1.00 ; \mathrm{TLI}=1.026 ; \mathrm{NFI}=.991 ; \mathrm{RMSEA}=.000$; SRMR $=.021)$. All the paths were statistically significant and the significance of indirect mediational paths was further confirmed using bootstrap resampling method. The model accounted for $18 \%$ of infertility-related stress and $41 \%$ of self-judgment variances.

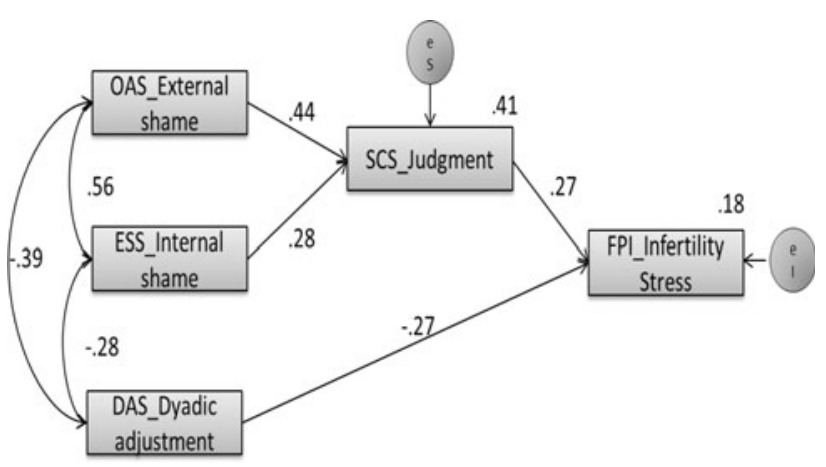

Fig. 3 Results of mediation path analysis showing the relationships among external shame (OAS), internal shame (ESS), dyadic adjustment (DAS) and infertility-related stress (FPI), having self-judgment (SCS_Judgment) as mediator in men, with standardised estimates and square multiple correlations $(n=147)$ 
Indirect mediational test results indicated that external shame (OAS) predicted greater infertility-related stress fully through increased self-judgment $\left(b_{\mathrm{OAS}}=.12,95 \%\right.$ $\mathrm{CI}=.046-.230)$. Internal shame (ESS) also predicted elevated infertility-related stress fully through greater selfjudgment (SCS_judgment) $\left(b_{\mathrm{ESS}}=.08,95 \% \mathrm{CI}=.021-\right.$ .143). Dyadic adjustment predicted diminished infertilityrelated stress directly with an effect of -.269 .

To summarize, in men self-judgment fully mediated the effect of external and internal shame on infertility-related stress. Dyadic adjustment had only a direct effect on infertility-related stress.

\section{Discussion}

The aim of the present study is to examine the role of external shame, internal shame, dyadic adjustment, selfcompassion and self-judgment in the prediction of infertility-related stress.

As expected, our participants presented higher mean scores in shame measures, self-judgment, and lower mean scores in self-compassion compared to those reported in fertile controls and community samples (Galhardo et al., 2011; Matos \& Pinto-Gouveia, 2010; Pinto-Gouveia et al., 2012). In line with previous findings (Galhardo et al., 2011; Moura-Ramos, Gameiro, Canavarro, Soares, et al., 2012; Neff, 2003a; Tangney \& Dearing, 2002), significant gender differences were found with women scoring higher than men in infertilityrelated stress, external shame, internal shame and self-judgment, but scoring lower in self-compassion. Contrary to other studies (Abedinia, Ramazanzadeh, \& Aghssa, 2003; Lee, Sun, \& Chao, 2001), no significant relationship was found between duration of infertility, diagnostic, or current medical treatment, and infertility-related stress in our sample.

In this study, scores on the dyadic adjustment were in accordance with the ones of previous studies which state that marital adjustment may be high in couples with infertility (Greil, 1997; Peterson, Newton, \& Rosen, 2003; Tuzer et al., 2010). This might be interpreted as a sign that couples tend to strengthen their relationship during an infertility process. Lack of differences in dyadic adjustment between men and women are in accordance with the current literature (Tuzer et al., 2010; Wright et al., 1991).

Bringing together our hypothesis and the findings on gender differences, we tested a mediator model in which selfcompassion and self-judgment were predicted as mediating the association between external shame, internal shame and dyadic adjustment, and infertility-related stress, in men and women with infertility. However, in men self-compassion was not a significant predictor and was excluded from the best fit model, whereas in women self-compassion emerged as a significant predictor in the best fit model.
In women, path analysis results revealed that external shame was a direct predictor of infertility-related stress, while the effects of internal shame on infertility-related stress were fully mediated by self-compassion. Furthermore, the effects of dyadic adjustment on infertility-related stress were partially mediated by self-compassion. In other words, in women, perceptions of the self as existing negatively in the minds of the others (i.e., external shame) have a direct impact on the perceived stress associated with infertility domains. Regarding internal shame, the impact of negative selfevaluations and feelings on distress related to infertility operates through self-compassion, which lessens the negative effect of such negative self-evaluations. It is worth noting that this suggests that having a warm and kind attitude towards oneself, perceiving one's inner experiences as part of a shared human condition and being able to distance oneself and observe one's private events (e.g., thoughts, feelings, memories, bodily sensations) seems to protect infertile women from the impact of a negative view of themselves, by attenuating their infertility-related stress. However, when these women believe they exist in the mind of others as someone with negative characteristics, a self-compassionate attitude offers no protective effect against the stress they may experience during their infertility process. As for dyadic adjustment, evaluating the couples' relationship in terms of marital satisfaction, cohesion, consensus and affective expression in a positive manner has a direct effect in diminishing the stress related to the infertility condition, but the negative impact of such evaluation on this stress also operates through increase in self-compassion attitudes. Interestingly, self-judgment, as the sum of self-criticism, isolation and over-identification (negative facets of selfcompassion) had no mediator role on these relationships in women with an infertility diagnosis.

In men, path analysis results indicated that external and internal shame predicted higher levels of infertility-related stress fully through greater self-judgment, while selfcompassion had no significant direct effect on the dependent variable. Dyadic adjustment directly predicted less infertility-related stress. These findings suggest that for men perceiving the self as being seen as inferior, inadequate, defective in the eyes of the others (i.e., external shame) and ones' own eyes (i.e., internal shame) seem to generate increased self-criticism, feelings of isolation, and over-identification with ones' inner experiences (i.e., selfjudgment), which in turn influences perceived stress related to infertility. Interestingly, it seems that in men, when negative facets of self-compassion are considered, the positive facets of this construct have no significant effect on these relationships. Furthermore, considering the marital relationship in a favorable manner (i.e., dyadic adjustment) is also important in explaining decreased stress related to the infertility diagnosis. 
Noteworthy, our results suggest that there is a distinct role of the positive and negative facets of self-compassion on the relationship between shame and infertility-related stress both in men and women. While in women the positive facets of self-compassion seem to have a protective effect on the impact of internal shame, in men the negative facets emerge as risk factors increasing the impact of externally and internally focused shame on infertilityrelated stress. Interestingly, in women beliefs about existing negatively in the minds of the others (i.e., external shame) have a direct effect on stress, while in men the effect of such beliefs operates through increased selfjudgment. These results add to the existing literature on the role of shame and self-compassion and self-judgment in patients with infertility (Galhardo et al., 2011; PintoGouveia et al., 2012) and are in line with current conceptualizations of shame and self-compassion (Gilbert, 2002, 2005; Neff, 2004; Neff et al., 2007; Tangney \& Dearing, 2002).

Nonetheless, our findings should be interpreted considering some methodological limitations. Although path analysis is a powerful statistical technique based on hypothetical relationships between variables established according to previous empirical and theoretical literature, our results rely on cross-sectional and self-report data. This design limits robust causal conclusions to be drawn from our results and points to the need of future replication studies with a longitudinal design, using other instruments such as semi-structured interviews. In addition, the use of a heterogeneous group of couples, at different stages of medical treatment, may add confounding variables which should be controlled in future research. Moreover, because of the low response rate, there is the possibility of individuals who did not take part in the study responding differently from those of the present sample.

Despite these methodological concerns, our findings may have some clinical implications. When working on psychological difficulties in patients with infertility it is important, not only to bear in mind the role of external and internal shame, which may function differently in men and women, but also the importance of addressing emotion regulation processes (self-compassion and self-judgment), which play a major role on the impact of shame on infertility-related stress and operate differently in men and women. These findings suggest that ACT (Hayes et al., 1999) and CompassionFocused Therapy (Gilbert, 2010a) may be adequate approaches for patients dealing with infertility. These third wave cognitive-behavioral therapies specifically target emotion regulation skills and may improve the effectiveness of psychotherapeutic interventions (Berking et al., 2008).

To sum up, key in this study was the finding that in women self-compassion mediates the effect of internal shame and dyadic adjustment on infertility-related stress, whereas in men self-judgment emerges as the mediator variable between external and internal shame and infertilityrelated stress. Although this research constitutes an exploratory analysis of these topics, this is the first study to investigate the mediating effects of self-compassion and self-judgment in the association between external shame, internal shame and dyadic adjustment and infertility-related stress in men and women separately.

Acknowledgments The authors extend their deepest thanks to all the couples who voluntarily participated in this study. The authors also wish to thank the infertility related public services and private clinics that collaborated in data collection and to the Portuguese Fertility Association for the support provided.

Funding This research has been supported by the first author Ph.D. Grant (SFRH/BD/68392/2010), sponsored by FCT (Portuguese Foundation for Science and Technology).

\section{References}

Abbey, A., Andrews, F. M., \& Halman, L. J. (1991). Gender's role in responses to infertility. Psychology of Women Quarterly, 15, 295-316. doi:10.1111/j.1471-6402.1991.tb00798.x.

Abedinia, N., Ramazanzadeh, F., \& Aghssa, M. M. (2003). Relationship between anxiety and depression with duration of pregnancy. Quartenaryy Journal Payesh, 4, 153-158.

Andrews, B., Qian, M., \& Valentine, J. (2002). Predicting depressive symptoms with a new measure of shame: The experience of shame scale. British Journal of Clinical Psychology, 41, 29-42.

Berking, M., Wupperman, P., Reichardt, A., Pejic, T., Dippel, A., \& Znoj, H. (2008). Emotion-regulation skills as a treatment target in psychotherapy. Behavior Research and Therapy, 46, 1230-1237. doi:10.1016/j.brat.2008.08.005.

Blackledge, J. T., \& Hayes, S. C. (2001). Emotion regulation in acceptance and commitment therapy [Review]. Journal of Clinical Psychology, 57, 243-255.

Boivin, J., \& Takefman, J. E. (1995). Stress level across stages of in vitro fertilization in subsequently pregnant and nonpregnant women. Fertility and Sterility, 64, 802-810.

Boivin, J., \& Takefman, J. E. (1996). Impact of the in vitro fertilization process on emotional, physical and relational variables. Human Reproduction, 11, 903-907.

Burns, L. H., \& Covington, S. N. (2006). Psychology of infertility. In S. N. Covington \& L. H. Burns (Eds.), Infertility counseling: A comprehensive handbook for clinicians. New York: Cambridge University Press.

Byrne, B. M. (2010). Structural equation modelling with AMOS: Basic concepts, applications, and programming (2nd ed.). New York: Routledge Academic.

Castilho, P., Pinto-Gouveia, J., \& Bento, E. (2011). Auto-compaixão: Estudo da validação da versão portuguesa da Escala da Autocompaixão e da sua relação com as experiências adversas na infância, a comparação social e a psicopatologia [Self-compassion: Validation study of the Portuguese version of the SelfCompassion Scale and its relationship with adverse experiences during childhood, social comparison and psychopathology]. Psychologica, 54, 203-230.

Chen, T. H., Chang, S. P., Tsai, C. F., \& Juang, K. D. (2004). Prevalence of depressive and anxiety disorders in an assisted reproductive technique clinic. Human Reproduction, 19, 2313-2318. 
Domar, A. D., Broome, A., Zuttermeister, P. C., Seibel, M., \& Friedman, R. (1992). The prevalence and predictability of depression in infertile women. Fertility and Sterility, 58, $1158-1163$.

Eugster, A., \& Vingerhoets, A. J. J. M. (1999). Psychological aspects of in vitro fertilization: A review. Social Science and Medicine, $48,575-589$.

Galhardo, A., Pinto-Gouveia, J., Cunha, M., \& Matos, M. (2011). The impact of shame and self-judgment on psychopathology in infertile patients. Human Reproduction, 26, 2408-2414.

Gilbert, P. (2002). Body shame: A biopsychosocial conceptualisation and overview, with treatment implications. In P. Gilbert \& J. Miles (Eds.), Body shame: Conceptualisation, research and treatment (pp. 3-54). London: Brunner.

Gilbert, P. (2005). Compassion: Conceptualisations, research and use in psychotherapy. London: Routledge.

Gilbert, P. (2010a). Compassion focused therapy. London: Routledge.

Gilbert, P. (2010b). The compassionate mind. London: Constable \& Robinson, Ltd.

Gilbert, P., \& Procter, S. (2006). Compassionate mind training for people with high shame and self-criticism: Overview and pilot study of a group therapy approach. Clinical Psychology and Psychotherapy, 13, 353-379. doi:10.1002/cpp.507.

Gomez, R., \& Leal, I. (2008). Ajustamento conjugal: Características psicométricas da versão portuguesa da Dyadic Adjustment Scale [Dyadic adjustment: Psychometric properties of the Portuguese version of the Dyadic Adjustment Scale]. Análise Psicológica, 4, $625-638$.

Goss, K., Gilbert, P., \& Allan, S. (1994). An exploration of shame measure: the 'others as shamer scale'. Personality and Individual Differences, 17, 713-717.

Greil, A. L. (1997). Infertility and psychological distress: A critical review of the literature. Social Science and Medicine, 45, 1679-1704. doi:S0277953697001020.

Guleç, G., Hassa, H., Gunes, E., \& Yenilmez, Ç. (2011). The effects of infertility on sexual functions and dyadic adjustment in couples that present for infertility treatment. Turkish Journal of Psychiatry, 22, 166-176.

Hayes, S. C., Stroshal, K. D., \& Wilson, K. G. (1999). Acceptance and commitment therapy: An experiential approach to behavior change. New York: The Guilford Press.

Irons, C., \& Gilbert, P. (2005). Evolved mechanisms in adolescent anxiety and depression symptoms: The role of attachment and social rank systems. Journal of Adolescence, 28, 325-341.

Kim, S., Thibodeau, R., \& Jorgensen, R. (2011). Shame, guilt, and depressive symptoms: A meta-analytic review. Psychological Bulletin, 137, 68-96.

Kline, R. B. (2005). Principles and practice of structural equation modelling (2nd ed.). New York: The Guilford Press.

Klonoff-Cohen, H., \& Natarajan, L. (2004). The Concerns During Assisted Reproductive Technologies (CART) scale and pregnancy outcomes. Fertility and Sterility, 81, 982-988. doi: 10.1016/j.fertnstert.2003.08.050.

Lee, T. Y., Sun, G. H., \& Chao, S. C. (2001). The effect of an infertility diagnosis on the distress, marital and sexual satisfaction between husbands and wives in Taiwan. Human Reproduction, 16, 1762-1767.

Mahajan, N. N., Turnbull, D. A., Davies, M. J., Jindal, U. N., Briggs, N. E., \& Taplin, J. E. (2010). Changes in affect and state anxiety across an in vitro fertilization/intracytoplasmic sperm injection cycle. Fertility and Sterility, 93, 517-526. doi:10.1016/ j.fertnstert.2008.12.054.

Matos, M., \& Pinto-Gouveia, J. (2010). Shame as a traumatic memory. Clinical Psychology \& Psychotherapy, 17, 299-312. doi:10.1002/cpp.659.
Menning, B. E. (1980). The emotional needs of infertile couples. Fertility and Sterility, 34, 313-319.

Monga, M., Alexandrescu, B., Katz, S. E., Stein, M., \& Ganiats, T. (2004). Impact of infertility on quality of life, marital adjustment, and sexual function. Urology, 63, 126-130.

Moura-Ramos, M., Gameiro, S., Canavarro, M. C., \& Soares, I. (2012a). Assessing infertility stress: Re-examining the factor structure of the Fertility Problem Inventory. Human Reproduction, 27, 496-505. doi:10.1093/humrep/der388.

Moura-Ramos, M., Gameiro, S., Canavarro, M. C., Soares, I., \& Santos, T. A. (2012b). The indirect effect of contextual factors on the emotional distress of infertile couples. Psychology \& Health, 27, 533-549. doi:10.1080/08870446.2011.598231.

Neff, K. D. (2003a). The development and validation of a scale to measure self-compassion. Self and Identity, 2, 223-250.

Neff, K. D. (2003b). Self-compassion: An alternative conceptualization of a healthy attitude toward oneself. Self and Identity, 2, 85-101. doi:10.1080/15298860390129863.

Neff, K. D. (2004). Self-compassion and psychological well-being. Constructivism in the Human Sciences, 9, 27-37.

Neff, K. D., Kirkpatrick, A., \& Rude, S. S. (2007). Self-compassion and adaptive psychological functioning. Journal of Research in Personality, 41, 139-154.

Newton, C. R., Sherrard, W., \& Glavac, I. (1999). The Fertility Problem Inventory: Measuring perceived infertility-related stress. Fertility and Sterility, 72, 54-62.

Pestana, M. H., \& Gageiro, J. N. (2005). Análise de dados para as ciências sociais: A complementaridade do SPSS [Data analises for social sciences: The SPSS complementarity]. Lisboa: Edições Sílabo.

Peterson, B. D., Newton, C. R., \& Rosen, K. H. (2003). Examining congruence between partners' perceived infertility-related stress and its relationship to marital adjustment and depression in infertile couples. Family Process, 42, 59-70.

Peterson, B. D., Newton, C. R., Rosen, K. H., \& Schulman, R. S. (2006). Coping processes of couples experiencing infertility. Family Relations, 55, 227-239.

Peterson, B. D., Pirritano, M., Block, J. M., \& Schmidt, L. (2011). Marital benefit and coping strategies in men and women undergoing unsuccessful fertility treatments over a 5-year period. [Multicenter Study Research Support, Non-U.S. Gov't]. Fertility and Sterility, 95, 1759-1763.e1. doi:10.1016/j.fertnstert. 2011.01.125.

Pinto-Gouveia, J., Galhardo, A., Cunha, M., \& Matos, M. (2012). Protective emotional regulation processes towards adjustment in infertile patients. Human Fertility, 15, 27-34. doi:10.3109/ 14647273.2011 .654310$.

Ramazanzadeh, F., Noorbala, A. A., Abedinia, N., \& Nazhizadeh, M. M. (2009). Emotional adjustment in infertile couples. Iranian Journal of Reproductive Medicine, 7, 97-103.

Schmidt, L., Holstein, B. E., Christensen, U., \& Boivin, J. (2005). Communication and coping as predictors of fertility problem stress: Cohort study of 816 participants who did not achieve a delivery after 12 months of fertility treatment. Human Reproduction, 20, 3248-3256.

Spanier, G. B. (1976). Measuring dyadic adjustment: New scales for assessing the quality of marriage and similar dyads. Journal of Marriage and the Family, 38, 15-28.

Tangney, J., \& Dearing, R. (2002). Shame and guilt. New York: The Guilford Press.

Tangney, J., Wagner, P., \& Gramzow, R. (1992). Proneness to shame, proneness to guilt and psychopathology. Journal of Abnormal Psychology, 101, 469-478.

Tuzer, V., Tuncel, A., Goka, S., Dogan-Bulut, S., Yursel, F. V., Atan, A., et al. (2010). Marital adjustment and emotional symptoms in 
infertile couples: Gender differences. Turkish Journal of Medical Science, 40, 229-237. doi:10.3906/sag-0901-17.

Verhaak, C. M., Lintsen, A. M., Evers, A. W., \& Braat, D. D. (2010). Who is at risk of emotional problems and how do you know? Screening of women going for IVF treatment. Human Reproduction, 25, 1234-1240. doi:10.1093/humrep/deq054.

Verhaak, C. M., \& Smeenk, J. M. J. (2007). Women's emotional adjustment to IVF: A systematic review of 25 years of research. Human Reproduction Update, 13, 27-36. doi:10.1093/humupd/ dm1040.

Volgsten, H., Skoog, S. A., Ekselius, L., Lundkvist, O., \& Sundstrom, P. I. (2010). Risk factors for psychiatric disorders in infertile women and men undergoing in vitro fertilization treatment. Fertility and Sterility, 93, 1088-1096. doi:10.1016/j.fertnstert. 2008.11.008
Wischmann, T., Stammer, H., Scherg, H., Gerhard, I., \& Verres, R. (2001). Psychosocial characteristics of infertile couples: A study by the 'Heidelberg Fertility Consultation Service'. Human Reproduction, 16, 1753-1761.

Wright, J., Duchesne, C., Sabourin, S., Bissonnette, F., Benoit, J., \& Girard, Y. (1991). Psychological distress and infertility: Men and women respond differently. Fertility and Sterility, 55, 100-108.

Zegers-Hochschild, F., Adamson, G. D., de Mouzon, J., Ishihara, O., Mansour, R., Nygren, K.,... Vanderpoel, S. (2009). International Committee for Monitoring Assisted Reproductive Technology (ICMART) and the World Health Organization (WHO) revised glossary of ART terminology, 2009. Fertility and Sterility, 92, 1520-1524. doi:10.1016/j.fertnstert.2009.09.009. 